\title{
Optical Nano-Imaging of Materials: Peeping Through Tip-Enhanced Raman Scattering ${ }^{\#}$
}

\author{
Satoshi Kawata ${ }^{* a, b}$ and Prabhat Verma ${ }^{a}$
}

\begin{abstract}
High-resolution optical nano-imaging, with a spatial resolution far beyond the diffraction limits of the probing light, has been shown by means of tip-enhanced near-field Raman spectroscopy. The capabilities of imaging the physical and chemical properties of materials have been demonstrated by imaging the diameter distribution of the nanotubes within an isolated bundle of single wall carbon nanotube. Further, by combining the nonlinear effects in the scattering process, it has been shown that the tip-enhanced coherent anti-Stokes Raman scattering imaging of DNA networks is capable of even higher spatial resolution and contrast.
\end{abstract}

Keywords: DNA networks · Optical nano-imaging · Nanotubes ·

Tip-enhanced coherent anti-Stokes Raman scattering (TE-CARS) · Tip-enhanced Raman spectroscopy (TERS)

\section{Introduction}

Optical microscopy has long been a wellestablished technique for the microanalysis in material sciences as well as in the biological sciences. However, the spatial resolution in such analyses is limited to half the probing wavelength, which is a few hundred nanometers for the visible light. This is the so-called diffraction limit, which is not small enough for imaging and studying nano-structures or molecular structures, and hence it leads us to the need for a technology that overcomes the diffraction limits. The basic idea to overcome the diffraction-limited resolution of conventional optical microscopy was experimentally demonstrated for visible light about two decades ago [1] by developing the technique of near-field scanning optical microscopy

\footnotetext{
${ }^{*}$ Correspondence: Prof. S. Kawata ${ }^{a, b}$

E-Mail: kawata@skawata.com

aDepartment of Applied Physics

Osaka University

Osaka, Japan

bRIKEN

Wako City

Saitama, Japan

\#Invited paper based on a contribution from the CEAC

'Nanoanalysis' Workshop at ETH Zürich, July 2006
}

(NSOM), which is a combination of the state-of-the-art near-field technology with conventional optical microscopy. The earlier NSOM experiments used aperture-type probes [2], however, later apertureless-type metal-coated probes were proposed [3] and ever since then the apertureless-type probe, such as the metal-coated cantilever tip of atomic force microscope (AFM) [4-11], has been utilized for achieving high resolution. The collective oscillation of free electrons in the metallized tip resonates with the probing light field, a phenomenon called localized surface plasmon polaritons, which couples with the electric field to provide the evanescent field at the tip apex [12]. Since the evanescent field is strongly localized near the tip, field-enhancement and superresolving capabilities are achieved. Ideally, it is possible to achieve molecular resolution with this technology.

If the NSOM technology is combined with the light scattering process, the enhanced evanescent field at the tip apex works as a nano light source, which provides enhanced scattering, making it possible to observe even very weak scattering modes at extremely high resolution. This is realized in tip-enhanced Raman scattering (TERS) [4-15], which is confocal Raman scattering combined with apertureless NSOM technology. The huge enhancement of Raman vibrational modes as well as high spatial resolution makes TERS one of the most powerful tools to identify the molecular compositions and inter- or intra-molecular bonds and to study nano-sized materials and biological samples with high resolution, especially for imaging.

In this article, we show some experimental results on the high-resolution imaging of carbon nanotubes, using TERS. Apart from the topographical information, we show that TERS imaging of an isolated bundle of single-wall carbon nanotube (SWNT) can also image the diameter distribution of the nanotubes within a bundle. Also, we demonstrate further improvement in the spatial resolution by combining nonlinear effects with TERS. This is done by tip-enhanced coherent anti-Stokes Raman scattering (TECARS), performed on the adenine sample, which is a DNA base. Apart from the high resolution, TE-CARS shows a much better signal-to-noise ratio, making it possible to image an adenine nanostructure by using weak phonon modes in coherent antiStokes Raman scattering (CARS) spectra.

\section{Experimental Setup}

The experimental setup for Raman measurements consisted of an inverted confocal microscope, a frequency-doubled $\mathrm{Nd}: \mathrm{YVO}_{4}$ laser $(\lambda=532 \mathrm{~nm})$, and a spectrometer fitted with an electronically cooled CCD detector. The incident light was focused onto the sample using a high-NA oil-immersion lens, and the scattered light was guided in the backscattering geometry through several optical elements, such as a notchfilter, pin-holes, and narrow slits, to the spectrometer. For TERS measurements, an 
AFM was added to the sample-stage on the optical microscope. The tip was prepared by evaporating silver (thickness $=30 \mathrm{~nm}$ ) on the commercial silicon cantilever used in AFM. The tip apex was observed using scanning electron microscope and it was found that the size of tip apex was about $30 \mathrm{~nm}$. For the precise controls of the tipposition and for distance-regulation in the near-field measurements, a piezo scanning stage of the AFM was mounted directly on the inverted microscope. The positioning of the tip on the sample surface was controlled with a split-photo-detector feedback system on the AFM. Raw Raman data were calibrated and treated to remove the unwanted background signals.

\section{TERS from Single-Wall Carbon Nanotubes}

We have demonstrated the applicability of tip-enhanced near-field Raman spectroscopy for imaging and analysis of local vibrational modes of an individual SWNT. Recently, far-field Raman spectroscopy has been recognized as a very powerful tool for SWNTs and achieved an individual SWNT detection [16] because much structural information including chirality and diameter can be deduced from the vibration modes [17]. To further detail the specific structuredependent 'single molecule' property, a single-molecule addressing capability at the nanometer scale is needed, which is now provided by the tip-enhanced near-field microscope. For these measurements, we sparsely spread out SWNTs on a coverslip, and focused the laser beam at a tube. Generally, our sample consisted of bundles of SWNTs, however, after spincasting, some individual SWNTs were separated out from aggregated bundles of SWNTs. Spectrum (a) in Fig. 1 shows a tip-enhanced near-field Raman spectrum of an individual SWNT, obtained by bringing a silver-coated nano-tip into close proximity to the sample. A remarkable tangential G-band mode at $1592 \mathrm{~cm}^{-1}$, representing the graphite mode of a SWNT, was observed. A small D-band peak, representing a defect mode, at $1331 \mathrm{~cm}^{-1}$ was also detected. For a comparison, a far-field spectrum was measured under exactly the same experimental conditions, except that the tip was moved away from the sample by 100 $\mu \mathrm{m}$. The corresponding spectrum is shown by spectrum (b) in Fig. 1, which shows that all Raman vibrations had almost vanished, except a small trace of the strong mode at $1592 \mathrm{~cm}^{-1}$. A comparison between the two spectra confirms the enhancement of Raman modes under the near-field configuration. The enhancement factor was estimated to be 1700 .

The radial breathing mode (RBM) of SWNTs is very sensitive to the diameter

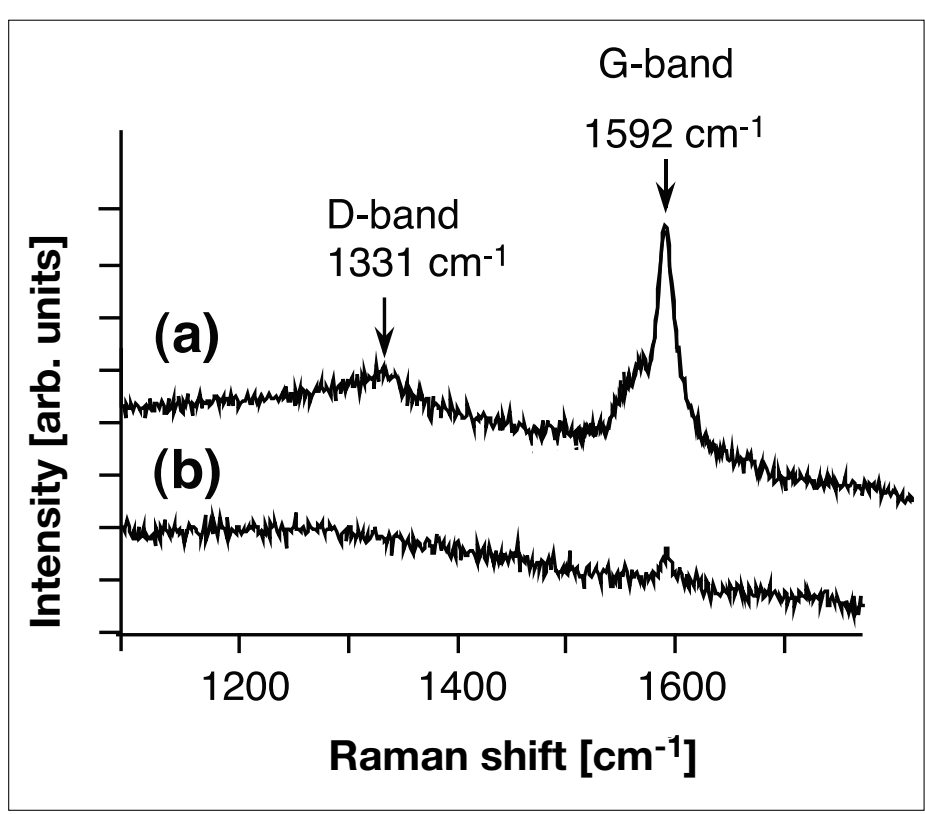

Fig. 1. Enhancement of D-band and G-band modes under the near-field configuration. (a) TERS spectrum when a metal-coated nano-tip was in contact with a single isolated SWNT, and (b) a corresponding far-field spectrum, when the tip was moved away from the SWNT.

of the nanotubes, as the frequency $\omega$ of the $\mathrm{RBM}$ is inversely proportional to the tube diameter, i.e. $d(\mathrm{~nm})=248 / \omega\left(\mathrm{cm}^{-1}\right)[18]$, and hence it can be used for the imaging of diameter distribution within an individual SWNT bundle. Fig. 2(a) shows an AFM image of an individual SWNT bundle obtained with a silver-coated tip, and the line profile corresponding to the dashed line is shown in Fig. 2(b). The TERS spectrum was measured by positioning the silver-coated tip at the location indicated by (i) in the image. As shown by the upper spectrum in Fig. 2(c), six peaks were clearly observed in the frequency region of the RBM, which correspond to the SWNTs with different diameters, present in the bundle. These peaks at 195, 222 , 236, 244, 278 and $299 \mathrm{~cm}^{-1}$ correspond to SWNTs of diameters $1.27,1.12,1.05,1.02$, 0.89 and $0.83 \mathrm{~nm}$, respectively. When the metallic tip was moved away by $100 \mu \mathrm{m}$ from the bundle (far-field), the Raman scattering signal from the bundle became too weak to be analyzed, as can be seen by the dashed spectrum in Fig. 2(c). Only a trace of the strongest peaks could be observed in the far-field spectrum, confirming that all the peaks observed in near-field spectrum were enhanced by the tip. The enhancement factor of the Raman scattering intensity for the radial breathing mode at $278 \mathrm{~cm}^{-1}$ was estimated to be about 200, while that for the Raman band at $236 \mathrm{~cm}^{-1}$ was estimated to be more than 1770 , with the assumption that the intensity of far field Raman scattering obtained without the tip is at most the standard deviation of the spectrum around the peak, because this peak was too weak to be distinguished from the background in the far-field spectrum. In the calculation of the enhancement factor, it was noted that the far-field component in the Raman signal comes from the whole bundle under the focal spot, while the near-field component originates only from the SWNTs present in the close vicinity of the tip apex.

Further, TERS spectra were measured by scanning the sample stage along the bundle, so that the tip position was at different locations indicated by (i)-(iv) in the AFM image, and the corresponding spectra are shown by (i)-(iv), respectively, in Fig. 2(c). These observation points were chosen at about $40 \mathrm{~nm}$ away from each other, which is far beyond the spatial resolution defined by the diffraction limits of the probing light. While the far-field spectra remain identical for all locations, the near-field spectra shown in Fig. 2(c) differ from one another. The variations in the peak intensities indicate that the diameter distribution within the bundle varies from point to point. For example, the near-field spectra (i) and (ii) show different intensity ratios between the peaks at 236 and $278 \mathrm{~cm}^{-1}$, or the spectrum (iii) shows a peak at $143 \mathrm{~cm}^{-1}$, which was not observed in other near-field spectra, indicating different diameter distributions at these three locations. The spectrum (iv) shows larger Raman intensities, suggesting that the density of the SWNTs is highest at that point. This bundle seems to contain SWNTs with diameters varying from 0.8 $\mathrm{nm}$ to $1.7 \mathrm{~nm}$, and different part of bundle contains different concentration of a particular diameter.

Since these TERS experiments were done under the contact-mode configuration of AFM, they involved a physical or mechanical interaction between the tip 

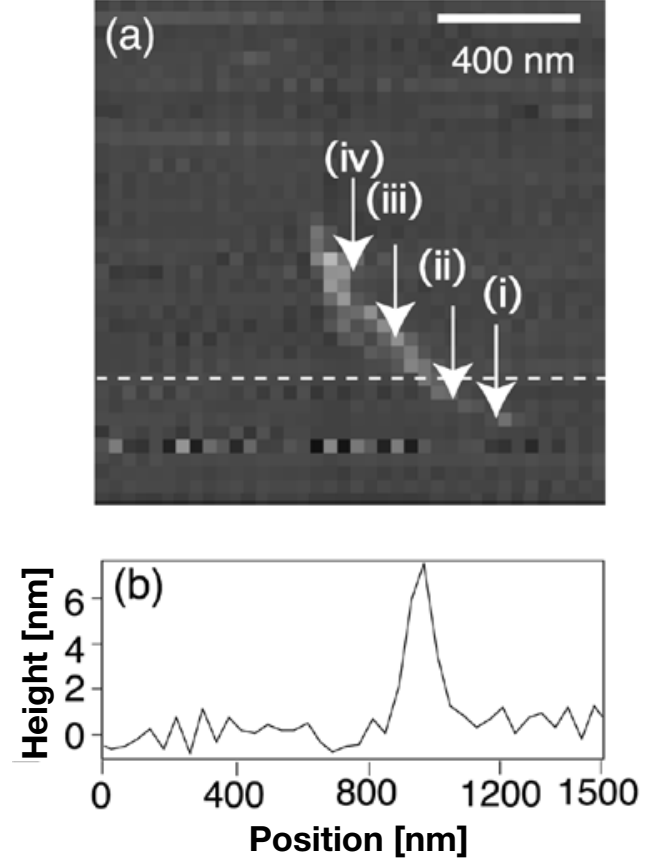

near field

far field

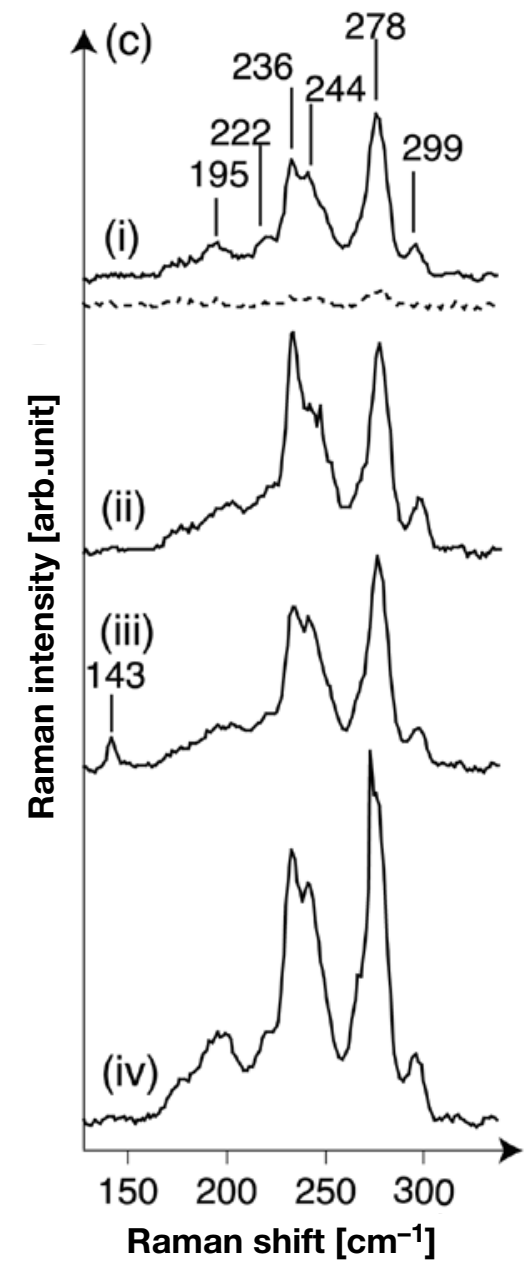

Fig. 2. (a) An AFM image of an individual SWNT bundle on the glass coverslip; (b) the line profile corresponding to the dashed line in the AFM image; (c) TERS spectra measured with tip positioned at the indicated points (i) to (iv) in the AFM image.
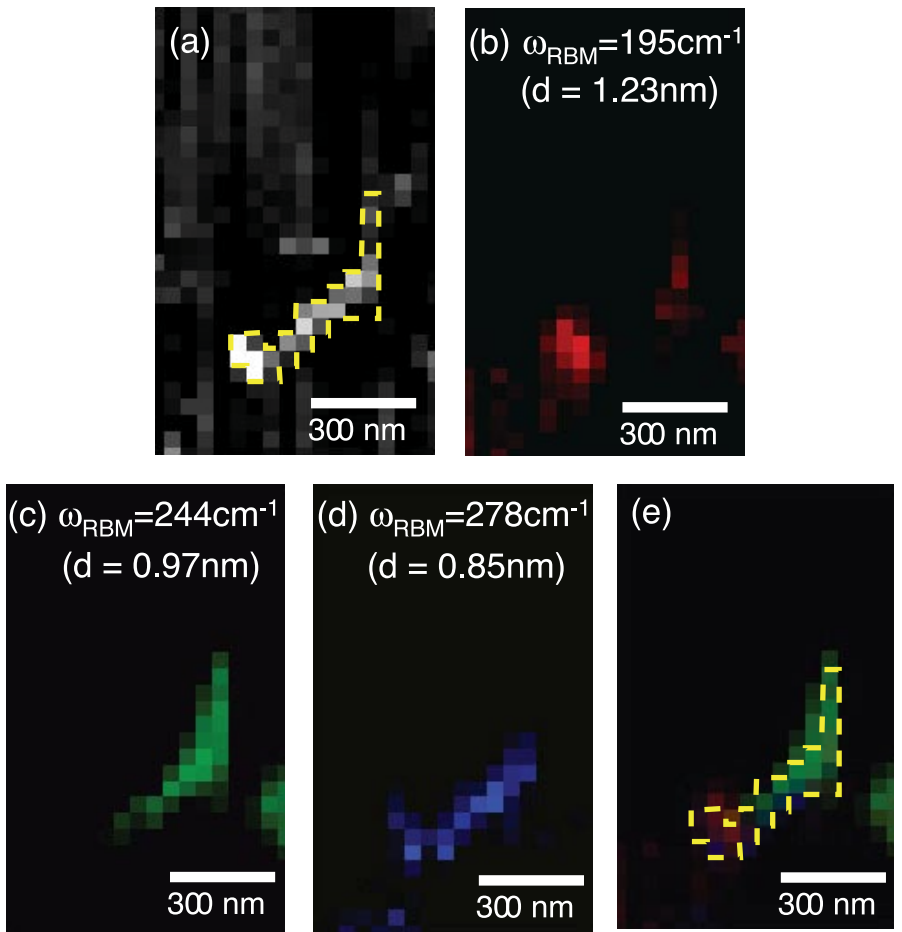

Fig. 3. (a) An AFM image of a SWNT bundle. TERS images obtained at (b) $195 \mathrm{~cm}^{-1}$ (c) $244 \mathrm{~cm}^{-1}$ and (d) $278 \mathrm{~cm}^{-1}$; (e) the mixed color image obtained by combining the three images in (b)-(d). and SWNTs. The tip can apply a force on SWNTs up to several nano-Newtons, which can distort the natural shape of the tube, and hence influence the Raman spectra. A systematic study on the influence of the tip-applied pressure in TERS experiments was reported recently [11]. It was noticed, however, that if the distance between the tip and the sample is optimized for the minimum amount of tip-applied pressure, then the force on a single SWNT is less than 0.1 $\mathrm{nN}$. Under this force, there were no noticeable changes in Raman spectra. In the present work, the distance between the tip and SWNTs was carefully optimized for the minimum pressure, and hence we believe that there was no significant influence of the tip-applied pressure on the Raman spectra of SWNTs, and hence on the diameter estimation.

\subsection{Imaging of Diameter Distribution within an Isolated Bundle of SWNT}

Fig. 2 demonstrates that the variation of diameter within an isolated bundle can be observed at a spatial resolution far beyond the diffraction limit of the probing light, which opens the possibilities of high-resolution diameter mapping through TERS within an isolated bundle. In order to image the diameter distribution within a bundle, a diameter-selective near-field Raman imaging of an isolated SWNT bundle was performed. This was done by fixing the spectrometer at three different frequencies of RBM observed in TERS spectra, and then scanning the whole sample for each pre-selected frequency. The obtained Raman images are shown in Fig. 3. The area surrounded by the dotted line in Fig. 3(a), which is a topographic image of the sample, indicates the bundle selected for this study. The spectrometer was fixed at frequencies $195 \mathrm{~cm}^{-1}$ (diameter $=1.23 \mathrm{~nm}), 244 \mathrm{~cm}^{-1}($ diameter $=0.97$ $\mathrm{nm})$ and $278 \mathrm{~cm}^{-1}($ diameter $=0.85 \mathrm{~nm})$, and the TERS images were obtained which are shown in Figs. 3(b)-(d), respectively. Fig. 3(b) reveals that the SWNTs having a diameter of $1.23 \mathrm{~nm}$ are localized at both edges of the bundle, Fig. 3(c) shows that the SWNTs with a diameter of $0.97 \mathrm{~nm}$ are prominently distributed towards the central and upper part of the bundle, and Fig. 3(d) indicates that the SWNTs with a diameter of $0.85 \mathrm{~nm}$ are mainly distributed towards the lower part of the bundle. Fig. 3(e) is a combination of Figs. 3(b)(d), which compares well with the topographic image shown in Fig. 3(a). Apart from the topographic image of the whole bundle, Fig. 3(e) also shows color-coded diameter distribution of the SWNTs within the bundle, which makes Fig. 3(e) much more informative than Fig. 3(a). This kind of imaging is not possible by conventional topographic imaging methods. 


\section{Imaging by Tip-Enhanced Nonlinear Spectroscopy}

Nonlinear optical effects contribute to spatial confinement of photons in a smaller volume than the size of the diffraction-limited focal spot. The intensity of the nonlinear effects is proportional to the high-order powers (square, cube, etc.) of the excitation light intensity. The spatial distribution of the signal emission (harmonic signal, two photon excited fluorescence, etc. ) becomes narrower than the intensity distribution of the excitation field. This leads to the reduction of the effective volume of light-matter interaction beyond the diffraction limit. Higher-order optical effects give much finer spatial response, as indicated in Fig. 4(a). The tip-enhanced near-field microscopy also benefits from the nonlinear effects. The volume of the light-matter interaction can be further confined to a tiny volume at the tip end due to the nonlinearity. Because of the nonlinear responses, even a small enhancement of the excitation field could lead to a huge enhancement of the emitted signal, allowing a reduction of the far-field background.

\subsection{Tip-Enhanced Coherent Anti- Stokes Raman Scattering}

In order to realize tip-enhanced nonlinear molecular-vibration spectroscopy, CARS spectroscopy is employed, which is one of the most widely used nonlinear Raman spectroscopies [19]. CARS is a fourwave mixing spectroscopic method, which includes a pump field $\left(\omega_{1}\right)$, a Stokes field $\left(\omega_{2} ; \omega_{2}<\omega_{1}\right)$, and a probe field $\left(\omega^{\prime}=\omega_{1}\right)$, and induces a non-linear polarization at the frequency of CARS, as shown in Fig. 4(b). When the frequency difference of $\omega_{1}$ and $\omega_{2}$, i.e. $\left(\omega_{1}-\omega_{2}\right)$, coincides with one of specific molecular vibrational frequencies of a given sample, the anti-Stokes Raman signal is resonantly generated. CARS spectroscopy is sensitive to molecular species and molecular conformation, resulting in a method of molecule identification without staining. As a CARS spectrum is obtained by scanning the frequency difference between two lasers, a high-resolution spectrometer is not required. Biological samples often have strong autofluorescence which overlaps with Stokes-shifted Raman scattering. Since the frequency of CARS is higher than those of the excitation lasers, CARS emission is separable from fluorescence. Although CARS spectroscopy has the above-mentioned advantages, it has not been utilized for microscopy since the report of CARS microscopy by Duncan et al. in 1982 [20]. Phase matching is required for CARS, and consequently it was thought to be difficult for high spatial resolution to coincide with the phase matching condition.

Recently several scientists have report-

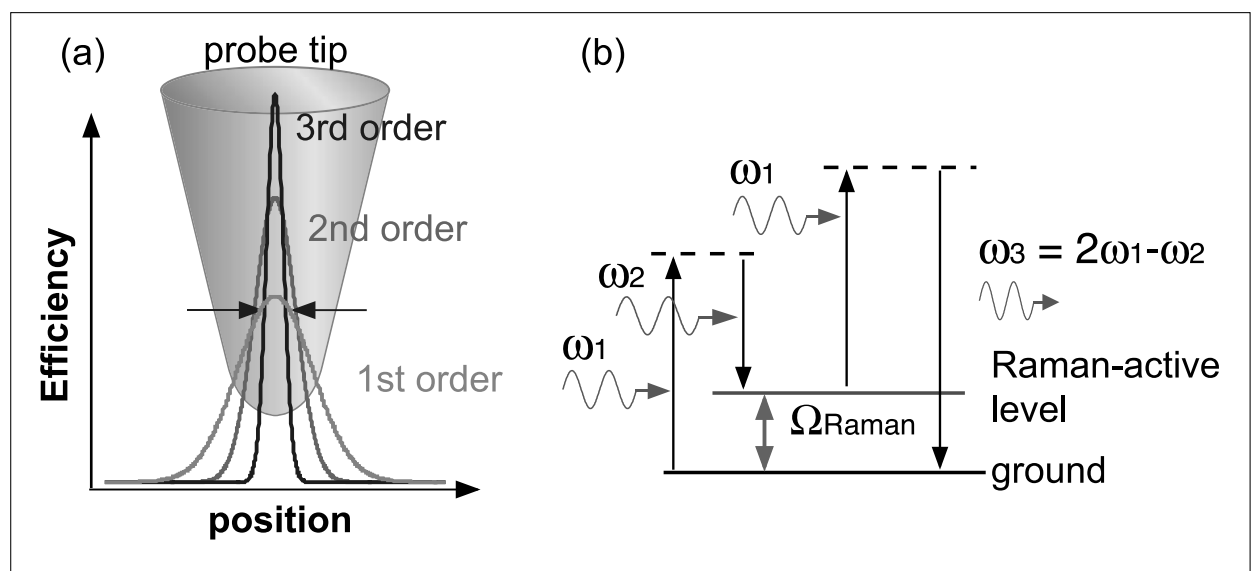

Fig. 4. (a) Spatial confinement of the excitation efficiency of high-order nonlinear effects. (b) Energy diagram for the CARS process. ed that tight focusing of the excitation fields with a high-NA objective lens can achieve CARS microscopy with three-dimensional imaging capability at a submicron scale [21][22]. The phase matching condition can be satisfied automatically in the focused fields of multiple angles [23]. In other words, it is not necessary that the phase matching condition be considered when the CARS polarizations are generated only in a volume smaller than the propagation wavelength of CARS light [24][25]. In our previous work, CARS was strongly amplified by isolated gold nanoparticles, which verified the possibility of the local enhancement of CARS by a metallic nano-structure [26]. Based on the concept mentioned above, one can observe CARS signals generated by the enhanced electric field at a metallic tip end of nanometric scale [27]. We have proposed a combination of the third-order nonlinear optical effect with the field enhancement effect of a metallic tip, that is, a technique for vibrational nano-imaging with TE-CARS. The alternative type of NSOM using an aperture-type probe was previously combined with CARS spectroscopy by Schaller et $a l$., where they employed a fiber probe for the signal collection of CARS excited by an external illumination and demonstrated chemical selective imaging of a biological specimen [21]. The use of tip-enhancement effects is, however, more advantageous with respect to spatial resolution, and is indispensable for the observation of small numbers of molecules. The backscattered component of the enhanced scattered light can be efficiently collected with the highNA focusing lens. By scanning the sample stage, while keeping the tip at the focused spot, one can acquire two-dimensional TE-CARS images of a specific vibrational mode with a high spatial resolution that is determined by the size of the tip end rather than the diffraction-limited focused spot.

\subsection{Experimental Setup for TE-CARS}

Fig. 5 shows the experimental system of TE-CARS microscopy [28][29]. Similar to TERS, the system mainly consists of an inverted microscope, an AFM using a silver-coated probe, and a monochromator. Two mode-locked Ti:sapphire lasers (pulse

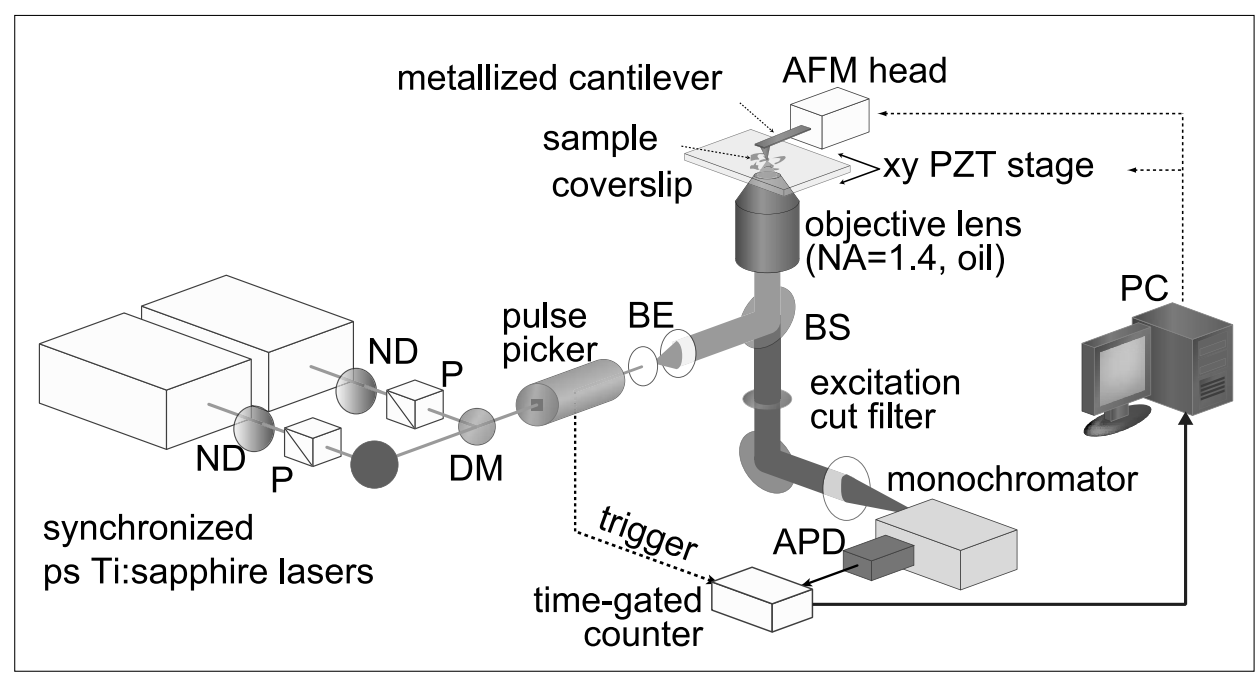

Fig. 5. A schematic for the experimental setup of TE-CARS microscopy 
duration: $5 \mathrm{ps}$, spectral band width: $4 \mathrm{~cm}^{-1}$, repetition rate: $80 \mathrm{MHz}$ ) were used for the excitation of CARS. The $\omega_{1}$ and $\omega_{2}$ beams were collinearly combined in time and space, and introduced into the microscope with an oil-immersion objective lens (NA = 1.4) focused onto the sample surface. The repetition rate of the excitation lasers was controlled by an electro-optically modulated pulse picker. The backscattered CARS emission enhanced by the probe tip was collected with the objective lens and detected with an avalanche-photodiode based photon-counting module through an excitationcut filter and the monochromator. The pulse signals from the APD were counted by a time-gated photon counter synchronously triggered with the pulse picker, which effectively reduces the dark counts down to almost 0 counts/sec.

\subsection{TE-CARS Imaging of DNA Clusters}

The DNA molecules of poly(dA-dT) aggregated into clusters were used as a model sample for CARS imaging. The poly(dAdT) solution in water $(250 \mu \mathrm{g} / \mathrm{ml})$ was cast and dried on a glass substrate at the room temperature with the fixation time of $24 \mathrm{~h}$. The dimensions of the clusters were typically $20 \mathrm{~nm}$ in height and $100 \mathrm{~nm}$ in width. The frequency difference of the two excitation lasers for CARS imaging was set to 1337 $\mathrm{cm}^{-1}$, corresponding to a Raman mode of adenine (ring-stretching mode of diazole) [8], by tuning the excitation frequencies $\omega_{1}$ and $\omega_{2}$ to be $12710 \mathrm{~cm}^{-1}\left(\omega_{1}: 786.77 \mathrm{~nm}\right)$ and $11373 \mathrm{~cm}^{-1}\left(\omega_{2}: 879.25 \mathrm{~nm}\right)$, respectively. After imaging in this resonant condition, the frequency of $\omega_{2}$ was changed such that the frequency difference corresponded to none of the Raman-active vibrations. Fig. 6 shows a spontaneous Stokes Raman spectrum of a DNA sample in the fingerprint region. The solid arrows on the spectrum denote the frequencies adopted for the 'on-resonant' and 'off-resonant' conditions in TE-CARS imaging.

Fig. 7 shows the CARS images of the DNA clusters obtained by our experimental system. Figs 7(a) and (b) are the TE-CARS image in the on-resonant condition $\left(\omega_{1}-\omega_{2}\right.$ $=1337 \mathrm{~cm}^{-1}$ ) and the simultaneously acquired topographic AFM image, respectively. The DNA clusters of about $100 \mathrm{~nm}$ diameter are visualized in Fig. 7(a). The two DNA clusters with separation of about $160 \mathrm{~nm}$ are clearly distinguished by the TE-CARS imaging. This indicates that the CARS imaging can successfully achieve the super-resolving capability beyond the diffraction limit of light. In the off-resonant condition $\left(\omega_{1}-\omega_{2}\right.$ $\left.=1278 \mathrm{~cm}^{-1}\right)$, the CARS signals mostly vanished in Fig. 7(c). Figs 7(a) and (c) verify that vibrationally resonant CARS is emitted from the DNA molecules at a specific frequency. However, a slight trace of the clusters could

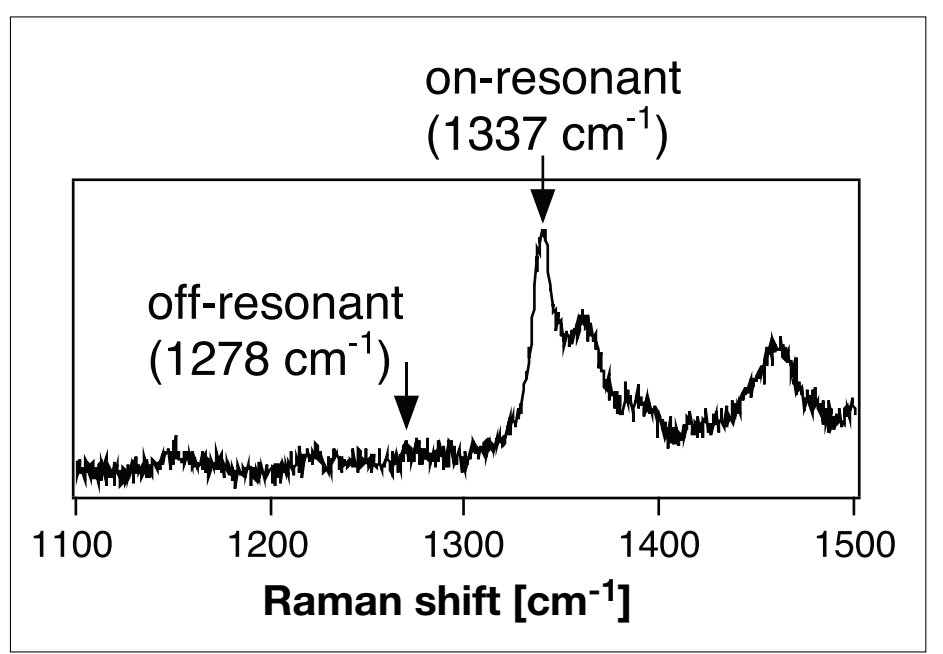

Fig. 6. A spontaneous Raman spectrum of DNA base. The two frequencies indicated by the arrows were selected for the off- and on-resonant TECARS imaging experiments.

be seen in the off-resonant condition in Fig. 7(d), which is the same as Fig. 7(c) but is shown with a different gray scale. This can be caused by both the frequency-invariant (non-resonant) component of the nonlinear susceptibility of DNA [19] and the topographic artifact [30]. Fig. 7(e) is a CARS image at the on-resonant frequency which was obtained after retracting the tip from the sample. The CARS signal was not detected in the CARS image without the silver tip, which confirms that the CARS polarization in Fig. 7(a) is effectively induced by the tipenhanced field. The background signal seen in Fig. 7(d) is attributed to both local fourwave-mixing emission of the silver probe due to its nonlinear susceptibility and to the white light continuum of silver due to electron-hole recombination [31][32]. These two components compete with the CARS process and degrade the image contrast and signal-tonoise ratio. Further, they subsequently limit the smallest number of molecules that can be observed. In this experiment, however, the TE-CARS signal intensity largely surpasses the background because the number of molecules in the excited volume is enough to induce the signal.

In order to assess the capability of the sensitivity of the TE-CARS microscopy, we prepared a DNA network of poly(dAdT)-poly(dA-dT) [33]. DNA (poly(dA$\mathrm{dT})$-poly(dA-dT) ) dissolved in water $(250$ $\mathrm{mg} / \mathrm{ml})$ was mixed with $\mathrm{MgCl}_{2}(0.5 \mathrm{mM})$ solution, then the DNA solution was cast on a coverslip and blow-dried after the fixation time of $2 \mathrm{~h}$. $\mathrm{Mg}^{2+}$ plays a role in the linkage between DNA and oxygen atoms of the glass surface. Fig. 8(a) shows a typical topographic image of the DNA network sample. The DNA network consists of bundles of DNA double-helix filaments aligned parallel on the glass substrate. Since the diameter of single DNA double-helix filaments is about
$2.5 \mathrm{~nm}$, the height of the bundle structures is $\sim 2.5 \mathrm{~nm}$, and the width is from $2.5 \mathrm{~nm}$ (for single filaments) to a few tens of nanometers (for $c a$. ten filaments). The TE-CARS images at the on- and off-resonant frequencies are shown in Figs. 8(b) and (c). The DNA bundles are observed at the resonant frequency in Fig. 8(b), while they cannot be visualized at the off-resonant frequency in Fig. 8(c). This indicates that the observed contrast is dominated by the vibrationally resonant CARS signals. Fig. 8(d) shows one-dimensional line profiles at $y=270 \mathrm{~nm}$ (corresponding to the position marked by horizontal arrows in the figure), which were acquired with steps of $\sim 5 \mathrm{~nm}$. The line profile of far-field CARS acquired without the silver tip is also added for comparison. Only the TE-CARS in the on-resonant condition has peaks at $x \sim 370 \mathrm{~nm}$ and $x \sim 700 \mathrm{~nm}$ where adenine molecules exist in the DNA double helix, while the other line profiles do not sense the existence of the molecules. The full width at half maxima of the peak at $x \sim$ $700 \mathrm{~nm}$ is $15 \mathrm{~nm}$, which confirms that with the existing TE-CARS setup, we are able to obtain a special resolution down to $15 \mathrm{~nm}$. This extremely high resolution is attributed to the combination of the near-field effects of TERS and the non-linearities of CARS. The intensity enhancement factor for each electric field is estimated to be $\sim 100$-fold. It should be noted that this enhancement factor of the field intensity already includes the nonlinear effects of CARS. The estimated value of the enhancement factor $(\sim 100)$ is quite realistic and reasonable, as compared to previous numerical results [34][35], although this estimation is very much subject to the changes in each parameter with highorder dependency. If this value of field enhancement is used for the estimation of the enhancement factor for TERS intensity, it would be about 10000 times (second 

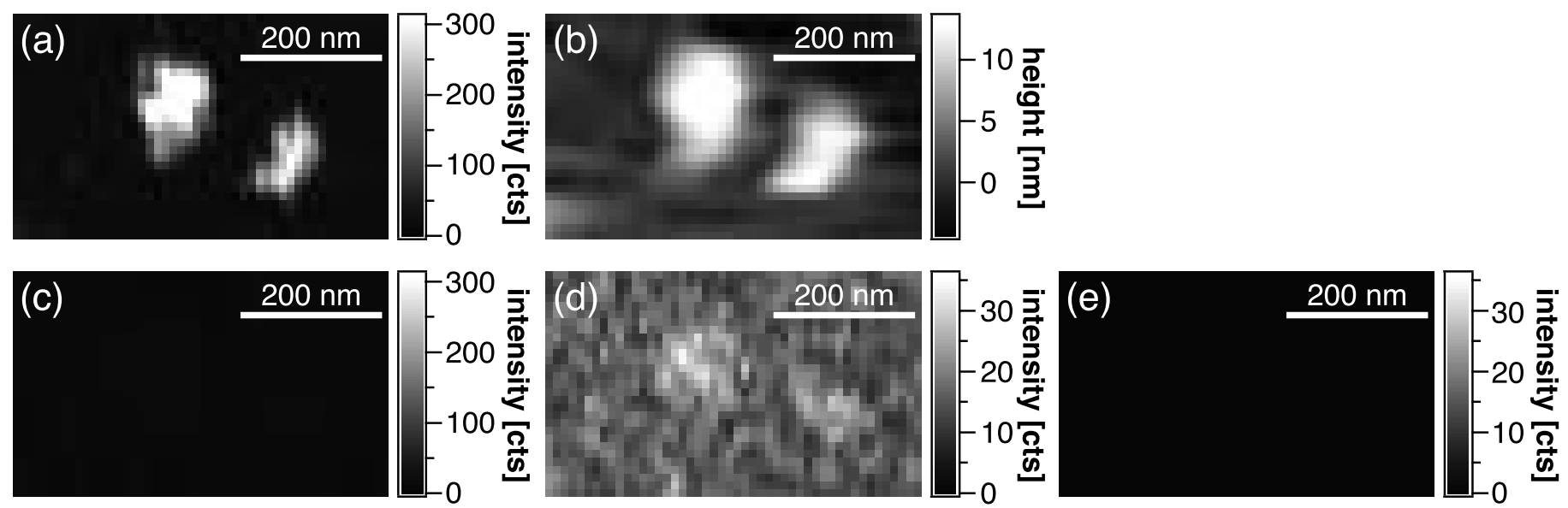

Fig. 7. CARS images of the DNA clusters. (a) Tip-enhanced CARS image at on-resonant frequency $\left(1337 \mathrm{~cm}^{-1}\right)$, and (b) the simultaneously obtained topographic image. (c) TE-CARS image at the off-resonant frequency $\left(1278 \mathrm{~cm}^{-1}\right)$. (d) The same image as (c) shown with a different gray scale. (e) CARS image of the corresponding area obtained without the silver tip. The scanned area was $500 \mathrm{~nm}$ by $300 \mathrm{~nm}$. The number of photons counted in $100 \mathrm{~ms}$ was recorded for one pixel. The acquisition time for one image was $3 \mathrm{~min}$. The average powers of the $\omega_{1}$ and $\omega_{2}$ beams were $30 \mu \mathrm{W}$ and $15 \mu \mathrm{W}$ at the $800 \mathrm{kHz}$ repetition rate.

power of the intensity enhancement). Our TERS results for SWNT sample, which showed an enhancement factor slightly less than 2000, somewhat agree with this order of enhancement. However, it should be noted here that the enhancement factors for TERS and TE-CARS experiments should not be directly compared, because these two experiments were done on two different samples with two different excitation wavelengths.

We also estimated the size of the locally excited volume of the DNA structure to be about 1 zeptoliter. The smallest detectable volume of DNA under the current experimental condition is estimated to be $1 / 4$ zeptoliter, which is derived from the signalto-noise ratio of 15:1 in Fig. 8(d) and the quadratic dependence of the CARS intensity on interaction volume. This indicates that our TE-CARS microscope is capable of sensing a vibrational-spectroscopic signal from an enormously small subzeptoliter volume.

\section{Conclusions}

A metal-coated nano-sized tip significantly improves Raman scattering spectroscopy to a novel analytical tool that provides important information about the sample, as well as enabling high-resolution optical imaging. Spatial resolution of molecular imaging reaches up to $15 \mathrm{~nm}$ by inducing nonlinear Raman scattering at the tip. If such tip effects work on a single molecule, there is the possibility that atomic resolution for chemical analysis and molecule imaging could be realized by

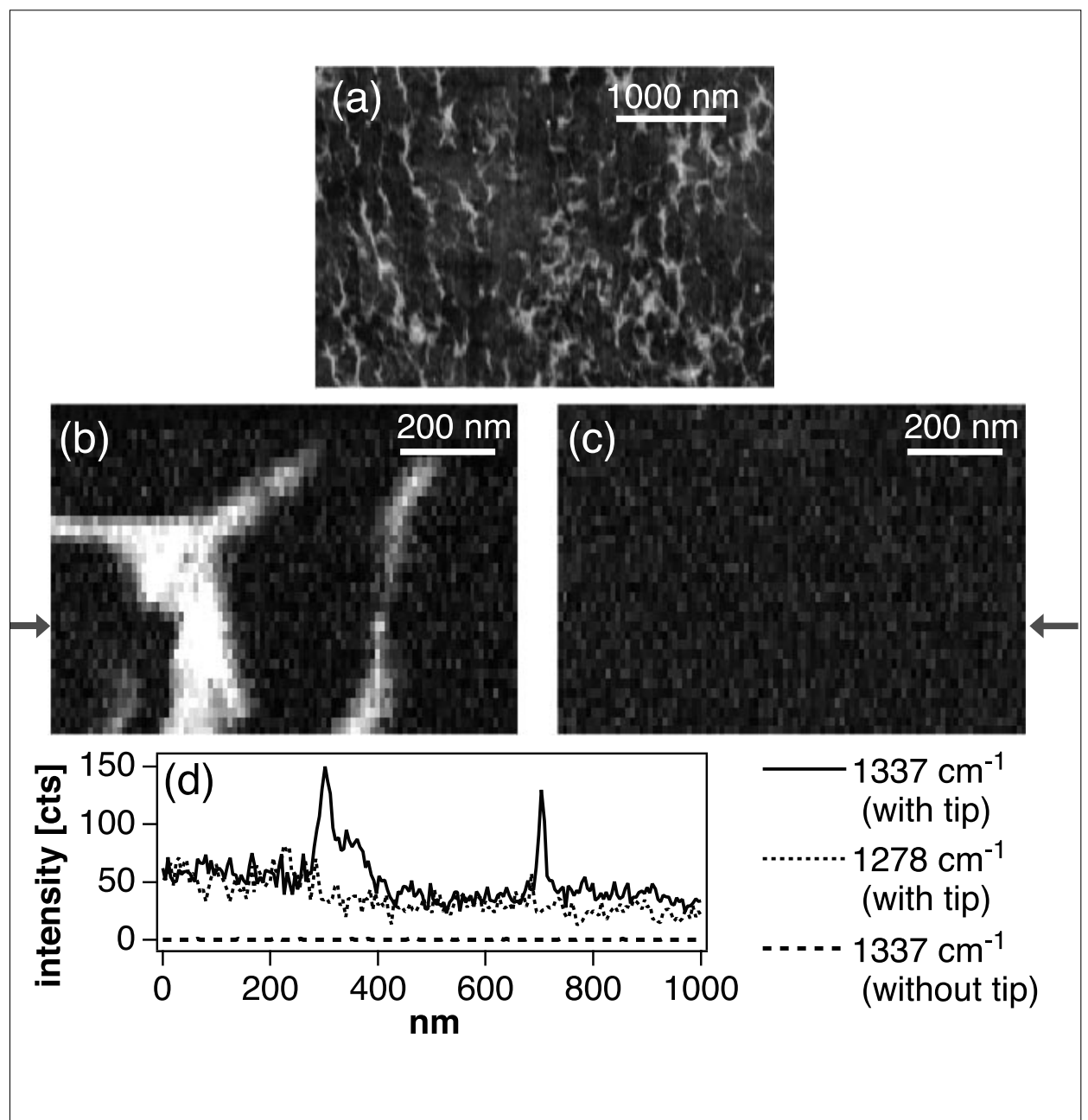

Fig. 8. TE-CARS images of the DNA network. (a) Topographic image of the DNA network. (b) TE-CARS image at on-resonant frequency $\left(1337 \mathrm{~cm}^{-1}\right)$. (c) TE-CARS image at the off-resonant frequency (1278 $\mathrm{cm}^{-1}$ ). (d) Cross sectional line profiles at $y=270 \mathrm{~nm}$ (indicated by the horizontal arrows). The scanned area is $1000 \mathrm{~nm}$ by $800 \mathrm{~nm}$. The number of photons counted in $100 \mathrm{~ms}$ was recorded for one pixel. The acquisition time for one image was $12 \mathrm{~min}$. The average powers of the $\omega_{1}$ and $\omega_{2}$ beams were 45 $\mu \mathrm{W}$ and $23 \mu \mathrm{W}$ at the $800 \mathrm{kHz}$ repetition rate. 
detecting Raman scattering of a perturbed molecule.

Received: September 13, 2006

[1] D.W. Pohl, W. Denk, M. Lanz, Appl. Phys. Lett. 1984, 44, 651.

[2] D.P. Tsai, A. Othonos, M. Moskovits, D. Uttamchandani, Appl. Phys. Lett. 1994, 64, 1768.

[3] Y. Inouye, S. Kawata, Opt. Lett. 1994, 19, 159.

[4] Y. Inouye, N. Hayazawa, K. Hayashi, Z. Sekkat, S. Kawata, Proc. SPIE 1999, 40.

[5] N. Hayazawa, Y. Inouye, Z. Sekkat, S. Kawata, Opt. Commun. 2000, 183, 333.

[6] N. Hayazawa, Y. Inouye, Z. Sekkat, S. Kawata, J. Chem. Phys. 2002, 117, 1296.

[7] N. Hayazawa, T. Yano, H. Watanabe, Y. Inouye, S. Kawata, Chem. Phys. Lett. 2003, 376, 174.

[8] H. Watanabe, Y. Ishida, N. Hayazawa, Y. Inouye, S. Kawata, Phys. Rev. B 2004, 69, 155418.

[9] P. Verma, K. Yamada, H. Watanabe, Y. Inouye S. Kawata, Phys. Rev. B 2006, 73, 045416.

[10] T. Yano, P. Verma, S. Kawata, Y. Inouye, Appl. Phys. Lett. 2006, 88, 093125.

[11] T. Yano, Y. Inouye, S. Kawata, Nano Lett. 2006, 6, 1269

[12] S. Kawata in 'Near-field Optics and surface plasmon polaritons', Ed. S. Kawata, Springer-Verlag, Berlin Heidelberg, 2001.

[13] M.S. Anderson, Appl. Phys. Lett. 2000, 76, 3130.

[14] R.M. Stöckle, Y.D. Suh, V. Deckert, R. Zenobi, Chem. Phys. Lett. 2000, 318, 131.

[15] A. Hartschuh, E.J. Sánchez, X.S. Xie, L. Novotny, Phys. Rev. Lett. 2003, 90, 095503.

[16] R.A. Crowell, E.L. Chronister, Chem. Phys. Lett. 1992, 195, 602.

[17] S.A. Hambir, J. Franken, D.E. Hare, E.L. Chronister, B.J. Baer, D.D. Dlott, J. Appl. Phys. 1997, 81, 2157.
[18] A. Jorio, R. Saito, J.H. Hafner, C.M. Lieber, M. Hunter, T. McClure, G. Dresselhaus, M.S. Dresselhaus, Phys. Rev. Lett. 2001, 86, 1118.

[19] Y.R. Shen, 'Principles of Nonlinear Optics', Wiley, New York, 1984.

[20] M.D. Duncan, J. Reintjes, T.J. Manuccia, Opt. Lett. 1982, 7, 350.

[21] R.D. Schaller, J. Ziegelbauer, L.F. Lee, L.H. Haber, R.J. Saykally, J. Phys. Chem. B 2002, 106, 8489.

[22] A. Zumbusch, G.R. Holtom, X.S. Xie, Phys. Rev. Lett. 1999, 82, 4142.

[23] M. Hashimoto, T. Araki, S. Kawata, Opt. Lett. 2000, 25, 1768.

[24] M. Hashimoto, T. Araki, J. Opt. Soc. Am. A 2001, 18, 771 .

[25] J.-X. Cheng, A. Volkmer, X.S. Xie, J. Opt. Soc. Am. B 2002, 19, 1363.

[26] Z. Xiaolin, R. Kopelman, Ultramicroscopy 1995, 61, 69 .

[27] T. Ichimura, N. Hayazawa, M. Hashimoto, Y. Inouye, S. Kawata, J. Raman Spectrosc. 2003, 34, 651 .

[28] T. Ichimura, N. Hayazawa, M. Hashimoto, Y. Inouye, S. Kawata, Phys. Rev. Lett. 2004, 92, 220801.

[29] T. Ichimura, N. Hayazawa, M. Hashimoto, Y. Inouye, S. Kawata, Appl. Phys. Lett. 2004, 84, 1768.

[30] B. Hecht, H. Bielefeldt, Y. Inouye, D.W. Pohl, L. Novotny, J. Appl. Phys. 1997, 81, 2492.

[31] G.T. Boyd, Z.H. Yu, Y.R. Shen, Phys. Rev B 1986, 33, 7923.

[32] J.P. Wilcoxon, J.E. Martin, J. Chem. Phys. 1998, $108,9137$.

[33] S. Tanaka, L.T. Cai, H. Tabata, T. Kawai, Jpn. J. Appl. Phys. 2001, 40, L407.

[34] H. Furukawa, S. Kawata, Opt. Commun. 1998, 148, 221.

[35] J.T. Krug II, E.J. Sánchez, X.S. Xie, J. Chem. Phys. 2002, 116, 10895. 